\title{
Performance Optimisation of Dual-Pump NALM Fibre Laser Using Machine Learning Inference
}

\author{
Ilya Gukov, ${ }^{1}$ Sonia Boscolo, ${ }^{2}$ Christophe Finot, ${ }^{3}$ and Sergei K. Turitsyn ${ }^{2,4}$ \\ ${ }^{1}$ Moscow Institute of Physics and Technology \& Skolkovo Institute of Science and Technology, Moscow, Russia \\ ${ }^{2}$ Aston Institute of Photonic Technologies, Aston University, Birmingham B4 7ET, United Kingdom \\ ${ }^{3}$ Laboratoire Interdisciplinaire CARNOT de Bourgogne, CNRS-Université de Bourgogne Franche-Comté, Dijon, France \\ ${ }^{4}$ Aston-NSU Joint Centre for Photonics, Novosibirsk State University, 630090, Novosibirsk, Russia \\ s.a.boscolo@aston.ac.uk
}

\begin{abstract}
We apply predictive regression to find optimum operating regimes in a recently proposed layout of a flexible Figure- 8 laser having two independently pumped segments of active fibre in its bidirectional ring.

OCIS codes: $190.4370,060.3510$.
\end{abstract}

\section{Introduction}

There are many mode-locked fibre configurations that are used to achieve pulsed operation. Here we consider one of the first reported schemes - the Figure-8 laser [1] - that is currently receiving renewed attention as a compact, reliable and robust, all-fibre short-pulse source, requiring only standard fibre components. Figure-8 lasers consist of a bidirectional and unidirectional ring. The bidirectional ring forms a loop mirror that is imbalanced (either actively or passively) to induce a differential phase, and consequently a power-dependent reflectivity that mimics the action of a saturable absorber, promoting pulse generation in the main laser cavity. While numerous parameters determine the performance of Figure8 lasers, once the laser is assembled, the reflectivity/transmittance of the loop mirror depends only on the intra-cavity radiation, and the only degree of freedom of the system is the amplifier pump power level. Although the latter can be adjusted over a relatively wide range in some configurations, this results in energy scaling at virtually constant peak power. This initially popular laser configuration lost its appeal for some time, probably because of the highly nonlinear and difficult to control intra-cavity radiation dynamics.

Recently, a new design of a Figure-8 laser employing a nonlinear amplifying loop mirror (NALM) with two segments of active fibre and two independently controlled pump power modules has been proposed and experimentally demonstrated $[2,3]$. This laser layout enables a wide range of adjustability of the peak power of the generated pulses, and provides access to a large variety of lasing regimes with adjustable power-independent pulse duration and spectrum width. In this paper, we numerically explore the broad space of operating states of the laser that can be accessed through independent control of the pump powers in the two gain segments. An additional, easy-to-control parameter that impacts the range of operating states of the laser is the coupling ratio of the laser output coupler. The general issue of reaching a target mode-locked laser regime with a setup featuring many adjustable parameters can be intelligently addressed by implementing machine learning strategies $[4,5]$. Here, we show that the dual-pump NALM laser performance can be efficiently optimised by deploying gradient boosting predictive regression for an automated exploration of the space of cavity parameters and rapid identification of high-energy mode-locked states.

\section{Machine learning for laser performance optimisation}

The setup [Fig. 1(a)] used in our model for generating data that characterises the laser consists of a passive unidirectional ring and a NALM connected via a 40/60 coupler. The NALM includes two sections of ytterbium-doped fibre, YDF1 and YDF2, of respective lengths $5.3 \mathrm{~m}$ and $5.0 \mathrm{~m}$, separated by a $12.4 \mathrm{~m}$-long section of single-mode normally dispersive fibre (NDF). The passive loop includes a $2.8 \mathrm{~m}$-long section of NDF and a $\beta /(1-\beta)$ fibre output coupler. We model the laser cavity by evolving the intra-cavity pulse dynamics in a component by component manner. In the fibres, propagation follows a standard modified nonlinear Schrödinger equation for the slowly-varying pulse envelope:

$$
i \psi_{z}-\frac{1}{2} \beta_{2} \psi_{t t}+\gamma|\psi|^{2} \psi=\frac{i}{2} g(z, \omega) \psi,
$$

where $\beta_{2}$ is the GVD parameter, $\gamma$ is the Kerr-nonlinearity coefficient, and $g$ is the gain coefficient. The latter is null for the passive fibres, considered lossless. In the YDFs, the gain $g$ includes saturation and spectral response, as $g(z, \omega)=$ $g_{m}(z) g_{p}(\omega)$. The gain coefficient amplitude $g_{m}(z)$ is dependent on the average signal and pump powers, the average power 


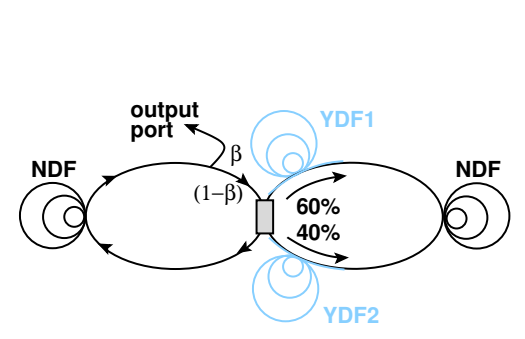

(a)

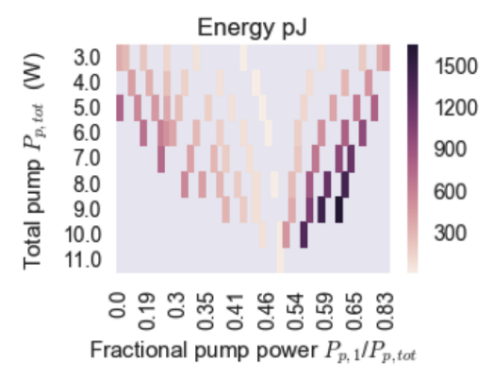

(b)

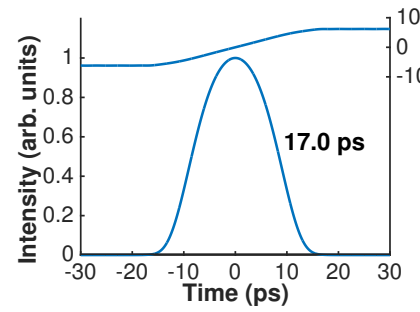

(c)

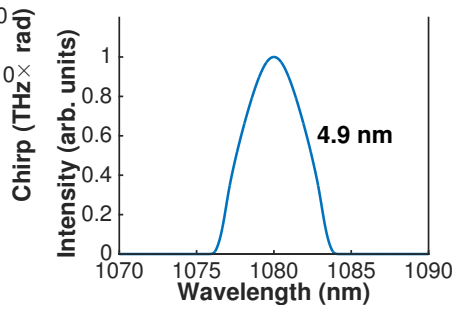

(d)

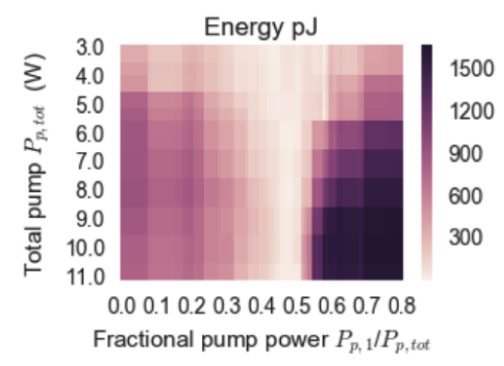

(e)

Fig. 1. (a) Laser schematic. (b) Map of output pulse energy values in the parameter space $\left(\left(P_{p, 1} / P_{p, \text { tot }}, P_{p \text {,tot }}\right)\right.$. (c) Temporal intensity and chirp profiles, and (d) spectral intensity profile of output pulse in the highest-energy mode-locked regime. (e) Output energy map generated by gradient boosting predictive regression.

dynamics being described by standard rate equations solved at each fibre longitudinal step. At a fixed output coupling factor $\beta$, stable, single pulses with different features - energy, peak power, pulse duration, spectral width, temporal and/or spectral shape - can be generated from the laser by varying the total pump level delivered to the active fibres, $P_{p, \text { tot }}=$ $P_{p, 1}+P_{p, 2}$, and the pump level difference between the two gain segments, $\left|P_{p, 1}-P_{p, 2}\right|$. A sample set of such mode-locked regimes found by simulation of the governing laser equations is shown in Fig. 1(b), which shows a map of output pulse energy values in the parameter space: fractional pump power, $P_{p, 1} / P_{p \text {,tot }}-$ total pump power, for $\beta=0.5$. The steadystate pulse characteristics of the highest-energy mode-locked regime are shown in Fig. 1(c)-(d). We have observed that an increase in pulse energy is mostly achieved owing to a combination of a peak power increase and pulse elongation in different proportions. Further, in general the higher the energy, the higher the chirp developed by the pulse.

We have trained a predictive regression model using a gradient boosted tree algorithm on our data to identify highenergy mode-locked solutions across the full range of $P_{p, 1} / P_{p, \text { tot }}$ and $P_{p, \text { tot }}$ variation. Fig. 1(e) shows the output energy map generated by the regression for $\beta=0.5$. The algorithm has instantly handled the whole parameter space with an absolute mean error of approximately $80 \mathrm{pJ}$. We note that the algorithm cannot detect multi-pulse mode-locking regimes, which are occasionally observed. Further, building of confidence intervals and boost strapping have showed that the data set used is too small in size to be statistically representative. Current work is considering the implementation of gradient boosting and neural network predictive regression methods on larger sets of sparse data in the three-dimensional parameter space of $\left(P_{p, 1} / P_{p, \text { tot }}, P_{p, \text { tot }}, \beta\right)$.

\section{Conclusion}

We have applied predictive regression to intelligently find optimum operating regimes in a novel layout of a flexible NALM laser with a second segment of active fibre and corresponding pump source. It is worth noting that this NALM layout enables reliable and reproducible live electronic adjustment of lasing regimes, which is practically impossible to do by adjusting fibre-based polarisation controllers in nonlinear-polarisation-evolution lasers.

\section{References}

1. I. N. Duling, "Subpicosecond all-fibre erbium laser," Electron. Lett. 27, 544 (1991).

2. A. Ivanenko et al., "Mode-locked long fibre master oscillator with intra-cavity power management and pulse energy $>12 \mu \mathrm{J}$," Opt. Express, 24, 6650 (2016).

3. S. Smirnov et al., "Layout of NALM fiber laser with adjustable peak power of generated pulses," Opt. Lett. 42, 1732 (2017).

4. T. Baumeister, S. L. Brunton, and J. N. Kutz, "Deep learning and model predictive control for self-tuning mode-locked lasers," J. Opt. Soc. Am. B 35, 617 (2018).

5. R. I. Woodward and E. J. R. Kelleher, “Towards 'smart lasers': self-optimisation of an ultrafast pulse source using a genetic algorithm,' Sci. Rep. 6, 37616 (2016). 